\title{
Bradykinesia in idiopathic normal pressure hydrocephalus evaluated by quantitative finger tapping test: preliminary study
}

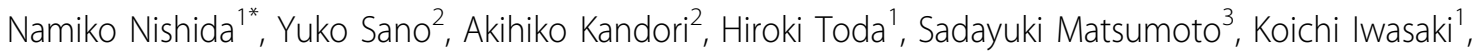 \\ Masatsune Ishikawa ${ }^{4}$
}

From Hydrocephalus 2015

Banff, Canada. 18-21 September 2015

\section{Introduction}

Movement disorders in idiopathic normal pressure hydrocephalus (iNPH) are represented by gait disturbance (i.e., lower body bradykinesia). However, upper extremity bradykinesia was frequently found among iNPH patients. We assessed their upper extremity function by quantitative finger tapping test and checked the correlation with other demographic factors.

\section{Methods}

We evaluated the 10-second finger tapping movements of 8 patients (age: $78.4 \pm 3.8$ y; males: 5 , females: 3 ) using magnetic-sensor coil system. Clinical symptoms were evaluated by the iNPH grading scale, mini-mental state examination and frontal assessment battery (FAB). The correlation of tapping parameters with clinical indicators was estimated.

\section{Results}

The patient's age correlated significantly with 6 of 21 finger-tapping parameters, including total tapping distance (Spearman $r=-0.82, p=0.013)$, coefficient of variation of maximum amplitude $(r=-0.78, p=0.023)$, energy balance $(r=-0.72, p=0.046)$, average maximum opening acceleration $(\mathrm{r}=-0.75, \mathrm{p}=0.034)$, tapping frequency $(\mathrm{r}=$ $-0.85, p=0.005)$, and average finger tapping interval $(r=$ $0.87, \mathrm{p}=0.007$ ). The severity of illness represented by iNPH grading scale correlated with other 2 parameters, including average maximum closing velocity $(r=-0.73$, $\mathrm{p}=0.043)$ and coefficient of variation of maximum closing velocity $(r=-0.79, \mathrm{p}=0.047)$.

\section{Conclusions}

Our data support the diagnostic value of quantitative finger tapping test for estimating the severity of bradykinesia underlying the iNPH symptomatology. Though preliminary, different patterns of correlations found in this study could potentially indicate the fundamental processes discriminating aging and disease progression.

\section{Authors' details \\ ${ }^{1}$ Department of Neurosurgery, Tazuke Kofukai Foundation, Medical Research Institute and Kitano Hospital, Japan. ${ }^{2}$ Hitachi Ltd. Research \& Development Group, Center for Technology Innovation - Healthcare, Japan. ${ }^{3}$ Department of Neurology, Tazuke Kofukai Foundation, Medical Research Institute and Kitano Hospital, Japan. ${ }^{4}$ Department of neurosurgery and normal pressure hydrocephalus center, Rakuwakai Otowa Hospital, Japan.}

Published: 18 September 2015

doi:10.1186/2045-8118-12-S1-P38

Cite this article as: Nishida et al:: Bradykinesia in idiopathic normal pressure hydrocephalus evaluated by quantitative finger tapping test: preliminary study. Fluids and Barriers of the CNS 2015 12(Suppl 1):P38.

\footnotetext{
* Correspondence: n-nishida@kitano-hp.or.jp

${ }^{1}$ Department of Neurosurgery, Tazuke Kofukai Foundation, Medical Research Institute and Kitano Hospital, Japan

Full list of author information is available at the end of the article
} 